\title{
Embedded Runge-Kutta scheme for step-size control in the Interaction Picture method
}

\author{
Stéphane Balac ${ }^{\mathrm{a}, \mathrm{b}}$, Fabrice Mahée,c \\ ${ }^{a} U E B$, Université Européenne de Bretagne, Université de Rennes 1 \\ ${ }^{b}$ CNRS UMR 6082 FOTON, Enssat, 6 rue de Kerampont, BP 80518, 22305 Lannion, France \\ ${ }^{c}$ CNRS UMR 6625 IRMAR, Campus de Beaulieu, 35042 Rennes, France
}

\begin{abstract}
When solving certain evolution type PDE such as the Schrödinger equation, the Interaction Picture method is a valuable alternative to Split-Step methods. The Interaction Picture method has good computational features when used together with the standard 4th order Runge-Kutta scheme (giving rise to the RK4-IP method). In this paper we present an embedded Runge-Kutta scheme with orders 3 and 4 with the aim to deliver an estimation of the local error for adaptive step-size control purposes in the Interaction Picture method. The corresponding ERK4(3)-IP method preserves the features of the RK4-IP method and provide a local error estimate at no significant extra cost.
\end{abstract}

Keywords: Interaction Picture method, Embedded Runge-Kutta method, Gross-Pitaevskii equation, Generalised non-linear Schrödinger equation, Split-Step method

2000 MSC: 78-04, 78M25, 65L06, 35Q60

\section{Introduction}

Recently a "fourth-order Runge-Kutta method in the interaction picture" method (RK4-IP method) has been proposed $[1,2]$ as a very promising alternative to the Split-Step methods for solving certain evolution type partial differential equations (PDE) such as the Gross-Pitaevskii equation (GPE) [3] or the generalised non-linear Schrödinger equation (GNLSE) [4]. The RK4-IP method has been developed in the 90's by the Bose-Einstein Condensate Theory Group of R. Ballagh from the Jack Dodd Centre at the University of Otago for solving the GPE in the context of Bose condensation. It was described in the Ph.D. thesis of B.M. Caradoc-Davies [1] and later in the Ph.D. thesis of M.J. Davis [2]. In this latter work an embedded Runge-Kutta (ERK) scheme was additionally used in conjunction with the RK4-IP method for adaptive step-size control purposes but without the expected efficiency. Since, the RK4-IP method has been widely used for numerical studies on BoseEinstein condensates, see e.g.[5-7].

The name "Interaction Picture" (IP) and the change of unknown at the heart of the method originate from quantum mechanics [8,9] where it is usual to choose an appropriate "picture" in which the physical properties of the studied system can be easily revealed and the calculation made simpler. In a numerical context the "Interaction Picture" approach is a way of solving certain PDE of evolution type by decoupling the linear and non-linear terms in the equation in order to make the resolution simpler. Typically, the solution to the PDE is obtained by solving a sequence of linear PDE problems and non-linear

Email addresses: stephane.balac@univ-rennes1.fr (Stéphane Balac), fabrice.mahe@univ-rennes1.fr (Fabrice Mahé) ordinary differential equations (ODE) in a given sequential order. This approach is very similar to the one used in Split-Step (SS) methods [10]. However while SS methods rely on an approximation formula such as the Strang formula [11] for the Symmetric Split-Step method, the IP method can be considered as exact since it amounts from a mathematical point of view to a change of unknown. In the IP method as in the SS method, the way the various terms in the PDE are "splitted" depends solely on a particular application and no general method is known. The motivation for using the IP or SS method for solving a PDE is essentially numerical; the numerical approximation of the solution to a linear PDE and to an ODE are easy, but a numerical integration involving all terms together is more difficult since CFL restrictions between time and spatial discretisation steps apply in order to guarantee stability of the numerical approximation scheme. A method of choice for solving ODE in the IP method is the fourth-order Runge-Kutta (RK4) method. Indeed compared to other RK methods it can be implemented in the context of the IP method to have very low memory consumption and high computational efficiency thanks to the advantageous position of the internal quadrature nodes of the RK4 scheme.

From a computational point of view the Interaction Picture method is very close to the Symmetric Split-Step method based on the Strang splitting formula widely used to solve the nonlinear Schrödinger equation [12, 13]. It has been for the first time applied for solving the GNLSE in optics by J. Hult in [4] where an experimental comparison of the RK4-IP method to other SS methods based on their numerical efficiency on benchmark problems in optics is presented. The experimental investigation undertaken in [4] indicates that the RK4-IP method exhibits interesting convergence properties and provides more accurate numerical results than comparable SS methods. This 
work on the RK4-IP for solving the GNLSE has come to our attention while working on the numerical simulation of incoherent optical wave propagation in non-linear fibers [14]. In [15] we have investigated the numerical properties of the RK4IP method and we have made a precise comparison between the RK4-IP method and the Symmetric Split-Step Fourier method with fourth-order Runge-Kutta scheme (S3F-RK4 method). We have shown that the RK4-IP method has a cost very same to the S3F-RK4 method due to a computational approach very similar to the one involved in the SS methods but has order of convergence 4 whereas the S3F-RK4 method is limited by the second order accuracy of the Strang splitting formula.

At present time a limitation of the RK4-IP method is the lack of a general automatic adaptive step-size control version of the method. Some attempts for using an adaptive step-size control strategy in conjunction with the RK4-IP method have however been made. The well known step-doubling approach (or Richardson extrapolation) is the more common way for local error estimation and step-size control [16]. However its computational over cost may be considered as prohibitive. An adaptive step-size control strategy based on a conservation energy principle has been proposed in [17] for the GNLSE. It is however only valid for lossless fibers. An embedded Runge-Kutta (ERK) method based on the Fehlberg formula [18, 19] has also been implemented in conjunction with the IP method in [2]. One major advantage of ERK methods for step-size control is that they are independent of the particular equation to be solved and therefore are very general. However the ease of implementation and the advantageous position of the internal quadrature nodes of the RK4 formula liable for the efficiency of the RK4IP method (by reducing both memory consumption and computational time) is lost with the Fehlberg formula and erode any benefit of the Fehlberg RK scheme for the IP method.

In this paper we present a 5 stage 3 rd order RK scheme embedding the standard 4th order RK scheme which possess the same features as the standard 4th order RK scheme when used in conjunction with the IP method. In particular this ERK4(3) scheme preserves the ease of implementation and the advantageous position of the internal quadrature nodes of the RK4 formula. Above all it delivers a local error estimate at no extra computational cost (at least when the current step is not rejected) for adaptive step-size control strategy.

The paper is organised as follows. In section 2 we present an overview of the IP method together with the standard 4th order RK method. In section 3 we recall the main features of ERK methods for local error estimation purposes. Section 4 is devoted to the building of an ERK scheme to obtain local error estimate in the IP method while preserving all the numerical advantages of the standard RK4 method. Last, in section 5 we present numerical simulation results in order to illustrate the features of the ERK4-IP method.

\section{Overview of the Interaction Picture method}

\subsection{PDE problem setting}

We first present a brief summary of the IP method for a general evolution equation in the form

$$
\frac{\partial}{\partial s} u(s, r)=\mathcal{D} u(s, r)+\mathcal{N}(u)(s, r)
$$

where $\mathcal{D}$ and $\mathcal{N}$ denote respectively linear and non-linear operators (that usually do not commute to each other); the linear differential operator $\mathcal{D}$ includes all the derivation terms with respect to the variable $r$ but does not involve derivation with respect to $s$ and the non-linear operator $\mathcal{N}$ does not involve derivation at all. This PDE is to be solved for the unknown $u$ in a set $I \times \Omega$ where typically $\Omega$ is an open subset in $\mathbb{R}^{d}, d \in \mathbb{N}^{*}$, and $I$ is an open interval in $\mathbb{R}$. Together with (1) we consider the initial condition: $u(s=0, r)=v_{0}(r), \forall r \in \Omega$ where $v_{0}$ is a sufficiently regular function from $\Omega$ to $\mathbb{C}$.

For instance, for the cubic non-linear Schrödinger equation

$$
\left\{\begin{array}{l}
\frac{\partial}{\partial t} u(t, \mathbf{r})+\mathrm{i} \Delta u(t, \mathbf{r})+\mathrm{i} \epsilon|u(t, \mathbf{r})|^{2} u(t, \mathbf{r})=0 \quad \forall \mathbf{r} \in \mathbb{R}^{2} \forall t \in \mathbb{R} \\
u(t=0, \mathbf{r})=u_{0}(\mathbf{r}) \quad \forall \mathbf{r} \in \mathbb{R}^{2}
\end{array}\right.
$$

where $\epsilon= \pm 1$ and $\Delta$ stands for the Laplacian operator in $\mathbb{R}^{2}$, we have $\mathcal{D}: u \mapsto \mathrm{i} \Delta u$ and $\mathcal{N}: u \mapsto \mathrm{i} \epsilon|u|^{2} u$.

For the GNLSE in optics $[14,20]$ we are interested in solving the following problem

$$
\left\{\begin{aligned}
\frac{\partial}{\partial z} A(z, t) & =\mathcal{D} A(z, t)+\mathcal{N}(A)(z, t) \quad \forall z \in] 0, L[\forall t \in \mathbb{R} \\
A(0, t) & =a_{0}(t) \quad \forall t \in \mathbb{R}
\end{aligned}\right.
$$

where the unknown $A$ corresponding to the slowly varying optical pulse envelope is a function of time $t$ and position $z$ along the fiber; the linear operator $\mathcal{D}$ is given by

$$
\mathcal{D}: A \mapsto-\frac{1}{2} \alpha A-\sum_{n=2}^{n_{\max }} \beta_{n} \frac{\mathrm{i}^{n-1}}{n !} \partial_{t}^{n} A
$$

where $\alpha$ is the linear attenuation coefficient and $\beta_{n}, n \geq 2$ are the linear dispersion coefficients; the non-linear operator $\mathcal{N}$ is given by

$$
\begin{aligned}
\mathcal{N}: A \mapsto \mathrm{i} \gamma & {\left[\mathrm{Id}+\frac{\mathrm{i}}{\omega_{0}} \frac{\partial}{\partial t}\right] \cdot\left(\left(1-f_{R}\right) A(z, t)|A(z, t)|^{2}\right.} \\
& \left.+f_{R} A(z, t) \int_{0}^{\infty} h_{R}(s)|A(z, t-s)|^{2} \mathrm{~d} s\right)
\end{aligned}
$$

where $h_{R}$ is the Raman time response function, $f_{R}$ represents the fractional contribution of the delayed Raman response to non-linear polarisation, $\gamma$ is the non-linear parameter and $\omega_{0}$ is the pulsation of the optical pulse assumed to be quasimonochromatic. We may notice that another splitting is possible for the GNLSE: the term $-\frac{1}{2} \alpha A$ can be added to the nonlinear operator $\mathcal{N}$ instead of the linear operator $\mathcal{D}$.

For the Gross-Pitaevskii (GP) equation used to explore the dynamics of vortexes in Bose-Einstein condensates in 2 or 3 
space dimensions [1-3], the condensate wave function $\psi$ is given in the domain $\Omega$ occupied by the condensate by

$$
\frac{\partial}{\partial t} \psi(\mathbf{r}, t)=\mathrm{i} \Delta \psi(\mathbf{r}, t)+\mathcal{N}(\psi)(\mathbf{r}, t)
$$

where $\Delta$ is the Laplacian operator in 2 or 3 dimensions, and

$$
\mathcal{N}: \psi \mapsto-\mathrm{i}\left(V \psi+C|\psi|^{2} \psi\right)
$$

where $V$ is the external potential applied (function of time $t$ and position $\mathbf{r}$ ) and $C$ is a constant proportional to the number of atoms in the condensate and to the scattering length. Equation (5) is to be solved to describe the condensate evolution from a given initial condensate state.

\subsection{The Interaction Picture method}

The IP method for solving (1) may be understood as follows. The interval $I=] 0, S$ [ is divided into $K$ sub-intervals where the grid points are denoted $s_{k}, k=\{0, \ldots, K\}$ such that $\left.] 0, S\right]=$ $\left.\left.\cup_{k=0}^{K-1}\right] s_{k}, s_{k+1}\right]$ where $0=s_{0}<s_{1}<\cdots<s_{K-1}<s_{K}=S$. For all $k \in\{0, \ldots, K-1\}$ the step length between $s_{k}$ and $s_{k+1}$ is denoted $h_{k}$ and we also set $s_{k+\frac{1}{2}}=s_{k}+\frac{h_{k}}{2}$.

Solving equation (1) for the initial condition $u(s=0, r)=$ $v_{0}(r), \forall r \in \Omega$ is equivalent to solving the following sequence of connected problems:

$$
\left\{\begin{aligned}
\frac{\partial}{\partial s} u_{0}(s, r) & \left.\left.=\mathcal{D} u_{0}(s, r)+\mathcal{N}\left(u_{0}\right)(s, r) \quad \forall s \in\right] s_{0}, s_{1}\right] \forall r \in \Omega \\
u_{0}\left(s_{0}, r\right) & =v_{0}(r) \quad \forall r \in \Omega
\end{aligned}\right.
$$

and $\forall k \in\{1, \ldots, K-1\}$

$$
\left\{\begin{aligned}
\frac{\partial}{\partial s} u_{k}(s, r) & \left.\left.=\mathcal{D} u_{k}(s, r)+\mathcal{N}\left(u_{k}\right)(s, r) \quad \forall s \in\right] s_{k}, s_{k+1}\right] \forall r \in \Omega \\
u_{k}\left(s_{k}, r\right) & =u_{k-1}\left(s_{k}, r\right) \quad \forall r \in \Omega
\end{aligned}\right.
$$

Obviously for all $k \in\{0, \ldots, K-1\}$ the unknown functions $u$ and $u_{k}$ are related by

$$
\forall s \in\left[s_{k}, s_{k+1}\right] \quad \forall r \in \Omega \quad u(s, r)=u_{k}(s, r) .
$$

Let us consider one of the problems defined in (7)-(8) for a given value of $k \in\{0, \ldots, K-1\}$. Such problem reads

$$
\left\{\begin{aligned}
\frac{\partial}{\partial s} u_{k}(s, r) & \left.\left.=\mathcal{D} u_{k}(s, r)+\mathcal{N}\left(u_{k}\right)(s, r) \quad \forall s \in\right] s_{k}, s_{k+1}\right] \forall r \in \Omega \\
u_{k}\left(s_{k}, r\right) & =v_{k}(r) \quad \forall r \in \Omega
\end{aligned}\right.
$$

where $v_{k}$ is a given function. We introduce as new unknown the mapping

$$
u_{k}^{\text {ip }}:(s, r) \in\left[s_{k}, s_{k+1}\right] \times \Omega \mapsto \exp \left(-\left(s-s_{k+\frac{1}{2}}\right) \mathcal{D}\right) \cdot u_{k}(s, r)
$$

where from a mathematical point of view the exponential terms have to be understood in the sense of the continuous group generated by the unbounded linear operator $\mathcal{D}$ [21]. From (9) one can show [15] that the new unknown $u_{k}^{\text {ip }}$ is solution to the following problem

$$
\left\{\begin{array}{c}
\left.\left.\frac{\partial}{\partial s} u_{k}^{\mathrm{ip}}(s, r)=\mathcal{G}_{k}\left(s, r, u_{k}^{\mathrm{ip}}(s, r)\right) \quad \forall s \in\right] s_{k}, s_{k+1}\right] \forall r \in \Omega \\
u_{k}^{\mathrm{ip}}\left(s_{k}, r\right)=\exp \left(-\left(s_{k}-s_{k+\frac{1}{2}}\right) \mathcal{D}\right) \cdot v_{k}(r) \quad \forall r \in \Omega
\end{array}\right.
$$

where $\mathcal{G}_{k}(s, r, \cdot)=\exp \left(-\left(s-s_{k+\frac{1}{2}}\right) \mathcal{D}\right) \circ \mathcal{N} \circ \exp \left(\left(s-s_{k+\frac{1}{2}}\right) \mathcal{D}\right)$. The major interest for using the change of unknown (10) is that on the contrary to problem (9), problem (11) for the unknown $u_{k}^{\text {ip }}$ does not anymore involve explicitly partial derivation with respect to the variable $r$. Partial derivation with respect to the variable $r$ now occurs through the operator $\exp \left( \pm\left(s-s_{k+\frac{1}{2}}\right) \mathcal{D}\right)$ which is computed separately. Thus problem (11) can be numerically solved just as if it was a non-linear ODE with $r$ as a parameter using a standard quadrature scheme for ODE such as Runge-Kutta (RK) methods.

\subsection{The RK4-IP method}

The "standard" Runge-Kutta (RK4) scheme is a method of choice to be used in conjunction with the IP method due to its convergence order 4 which offers a good compromise between accuracy of the result and cost of the computations. Besides the values of its internal quadrature nodes $(0,1 / 2,1)$ imply by symmetry some cancellations in the terms to be computed compared to other 4th order RK schemes when used in conjunction with the IP method, reducing the global computational cost of the method. The standard RK4 method is defined by the following Butcher tableau [18]

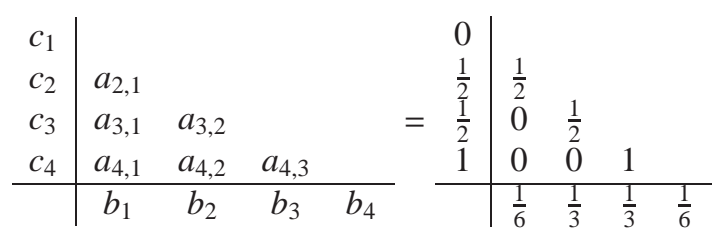

One step of the RK4 scheme defined by (12) is used to approach the solution to problem (11) for all $k \in\{0, \ldots, K-1\}$ as follows: $\forall r \in \Omega$

$$
u_{k}^{\mathrm{ip}}\left(s_{k+1}, r\right) \approx u_{k}^{\mathrm{ip}}\left(s_{k}, r\right)+\frac{h_{k}}{6}\left(\alpha_{1}+2 \alpha_{2}+2 \alpha_{3}+\alpha_{4}\right)
$$

where

$$
\begin{aligned}
\alpha_{1} & =\mathcal{G}_{k}\left(s_{k}, r, u_{k}^{\mathrm{ip}}\left(s_{k}, r\right)\right)=\exp \left(\frac{h_{k}}{2} \mathcal{D}\right) \cdot \mathcal{N}\left(\exp \left(-\frac{h_{k}}{2} \mathcal{D}\right) \cdot u_{k}^{\mathrm{ip}}\left(s_{k}, r\right)\right) \\
\alpha_{2} & =\mathcal{G}_{k}\left(s_{k}+\frac{h_{k}}{2}, r, u_{k}^{\mathrm{ip}}\left(s_{k}, r\right)+\frac{h_{k}}{2} \alpha_{1}\right)=\mathcal{N}\left(u_{k}^{\mathrm{ip}}\left(s_{k}, r\right)+\frac{h_{k}}{2} \alpha_{1}\right) \\
\alpha_{3} & =\mathcal{G}_{k}\left(s_{k}+\frac{h_{k}}{2}, r, u_{k}^{\mathrm{ip}}\left(s_{k}, r\right)+\frac{h_{k}}{2} \alpha_{2}\right)=\mathcal{N}\left(u_{k}^{\mathrm{ip}}\left(s_{k}, r\right)+\frac{h_{k}}{2} \alpha_{2}\right) \\
\alpha_{4} & =\mathcal{G}_{k}\left(s_{k}+h_{k}, r, u_{k}^{\mathrm{ip}}\left(s_{k}, r\right)+h_{k} \alpha_{3}\right) \\
& =\exp \left(-\frac{h_{k}}{2} \mathcal{D}\right) \cdot \mathcal{N}\left(\exp \left(\frac{h_{k}}{2} \mathcal{D}\right) \cdot\left[u_{k}^{\mathrm{ip}}\left(s_{k}, r\right)+h_{k} \alpha_{3}\right]\right) .
\end{aligned}
$$

From the change of unknown (10) the solution to problem (9) is approximated $\forall r \in \Omega$ as $u_{k}\left(s_{k+1}, r\right) \approx v_{k+1}(r)$ where

$$
v_{k+1}(r)=\exp \left(\frac{h_{k}}{2} \mathcal{D}\right) \cdot\left[u_{k}^{\mathrm{ip}}\left(s_{k}, r\right)+\frac{h_{k}}{6}\left(\alpha_{1}+2 \alpha_{2}+2 \alpha_{3}+\alpha_{4}\right)\right] .
$$


Actually since we are interested in computing $v_{k+1}(r)$ rather than $u_{k}^{\text {ip }}\left(s_{k+1}, r\right)$ which is a go-between in the computational approach, we recast the above approximation scheme for all $k \in\{0, \ldots, K-1\}$ in the following way to reduce its computational cost. We set

$$
\begin{aligned}
v_{k}^{\mathrm{ip}}(r) & =\exp \left(\frac{h}{2} \mathcal{D}\right) \cdot v_{k}(r) \\
\alpha_{1} & =\exp \left(\frac{h}{2} \mathcal{D}\right) \cdot \mathcal{N}\left(v_{k}(r)\right) \\
\alpha_{2} & =\mathcal{N}\left(v_{k}^{\mathrm{ip}}+\frac{h_{k}}{2} \alpha_{1}\right) \\
\alpha_{3} & =\mathcal{N}\left(v_{k}^{\mathrm{ip}}+\frac{h_{k}}{2} \alpha_{2}\right) \\
\alpha_{4}^{\prime} & =\mathcal{N}\left(\exp \left(\frac{h_{k}}{2} \mathcal{D}\right) \cdot\left[v_{k}^{\mathrm{ip}}+h_{k} \alpha_{3}\right]\right)
\end{aligned}
$$

and

$$
v_{k+1}(r)=\exp \left(\frac{h_{k}}{2} \mathcal{D}\right) \cdot\left(v_{k}^{\mathrm{ip}}(r)+\frac{h_{k}}{6}\left(\alpha_{1}+2 \alpha_{2}+2 \alpha_{3}\right)\right)+\frac{h_{k}}{6} \alpha_{4}^{\prime} .
$$

Of course, the crucial point in the above computational scheme - referred as the RK4-IP method - is the way the 4 mappings involving the $\exp \left(\frac{h_{k}}{2} \cdot \mathcal{D}\right)$ operator are computed. For a regular function $\varphi$, the mapping $r \mapsto \exp \left(\frac{h_{k}}{2} \mathcal{D}\right) \cdot \varphi(r)$ coincides with the solution for $s=s_{k+\frac{1}{2}}$ to the following linear evolution problem [15]

$$
\left\{\begin{aligned}
\frac{\partial}{\partial s} w(s, r) & \left.=\mathcal{D} w(s, r) \quad \forall s \in] s_{k}, s_{k+\frac{1}{2}}\right] \forall r \in \Omega \\
w\left(s_{k}, r\right) & =\varphi(r) \quad \forall r \in \Omega
\end{aligned}\right.
$$

In each step $k$ a major part of the computational effort lies in the resolution of this linear PDE problem for 4 distinct initial conditions. The numerical method used to solve it is strongly dependent to the linear operator $\mathcal{D}$ and domain $\Omega$, that is to say to the physical application under consideration. For the GPE e.g. this PDE problem is a heat type problem set in a $2 \mathrm{D}$ or 3D domain. In [1-3] it is solved by a Fourier spectral method. For the GNLSE, problem (16) where $\Omega=\mathbb{R}$ can be solved by a direct use of Fourier transforms [4, 14]. Moreover the cost of the evaluation of the 4 non-linear terms $\mathcal{N}(\varphi)$ is strongly dependent to the physical application. Nevertheless it is a direct function evaluation without intermediate PDE problem to be solved. Thus, in designing a new ERK method for adaptive step-size control purposes we have to keep in mind that the global computational cost of the method will be directly proportional to the number of exponential operators $\exp \left(\frac{h_{k}}{2} \mathcal{D}\right)$ and to a lower degree to the number of non-linear operators $\mathcal{N}$ involved.

Finally, an important point to be noticed concerns the values of the coefficients $c_{1}=0, c_{2}=\frac{1}{2}, c_{3}=\frac{1}{2}$ and $c_{4}=1$ of the RK4 scheme (12) in the efficiency of the RK4-IP method. Indeed, in conjunction with the choice of $s_{k+\frac{1}{2}}=s_{k}+\frac{h_{k}}{2}$ in the change of unknown (10), this particular choice of the $c_{i}$ coefficients enables the cancellation of 4 exponential operator terms in (15) compared to other possible sets of values, and therefore save up an important computational cost.

\section{Local error estimate by using a 4th order embedded Runge-Kutta method}

\subsection{Overview of embedded Runge-Kutta methods}

Embedded Runge-Kutta (ERK) methods are special RK methods designed to deliver two approximations of the solu- tion of the ODE under consideration, corresponding to 2 RK schemes of different convergence orders $p$ and $q(q>p)$. These 2 approximations of the solution can be considered as an accurate approximate solution (the one computed with the numerical scheme of higher order $q$ ) and a coarse approximate solution (the one computed with the one of lower order $p$ ). These 2 approximate solutions obtained with RK schemes of different orders can be combined in a specific way so as to deliver an estimation of the local error committed while approaching the solution with the lower order method.

Assuming that for problem (11) the solution value at grid point $s_{k}$ is regarded as exact (because we are concerned by an estimation of the local error), we denote by $v_{k+1}^{i p}\left(\operatorname{resp} . \widetilde{v}_{k+1}^{i p}\right)$ the coarse (resp. accurate) approximate solution at grid point $s_{k+1}$ given by the $p$-th order RK (resp. $q$-th order RK) scheme. The local errors for each of the 2 methods are respectively given by [18]

$$
\begin{aligned}
& \ell_{k+1}^{[p]}=u^{i p}\left(s_{k+1}, r\right)-v_{k+1}^{i p}(r)=\psi_{p}\left(s_{k}, r, v_{k}^{i p}\right) h_{k}^{p+1}+\varnothing\left(h_{k}^{p+2}\right) \\
& \ell_{k+1}^{[q]}=u^{i p}\left(s_{k+1}, r\right)-\widetilde{v}_{k+1}^{i p}(r)=\psi_{q}\left(s_{k}, r, v_{k}^{i p}\right) h_{k}^{q+1}+\varnothing\left(h_{k}^{q+2}\right)
\end{aligned}
$$

where $\psi_{p}$ (resp. $\psi_{q}$ ) is a function of the elementary differentials of order $p$ (resp. $q$ ) of $u^{i p}$. By difference of this 2 relations we obtain

$$
\widetilde{v}_{k+1}^{i p}(r)-v_{k+1}^{i p}(r)=\psi_{p}\left(s_{k}, r, v_{k}^{i p}\right) h_{k}^{p+1}+\varnothing\left(h_{k}^{p+2}\right) .
$$

Thus the local error for the RK scheme of lower order at grid point $s_{k}$ can be approximated, with an error in $\varnothing\left(h_{k}^{p+2}\right)$, in the following way

$$
\ell_{k+1}^{[p]}=\psi_{p}\left(s_{k}, r, v_{k}^{i p}\right) h_{k}^{p+1}+\varnothing\left(h_{k}^{p+2}\right) \approx \widetilde{v}_{k+1}^{i p}(r)-v_{k+1}^{i p}(r) .
$$

In general, ERK methods are constructed with $q=p+1$. One of the most famous ERK method is the Fehlberg 4(5) [18, 19]. It has 6 stages and delivers a RK approximation of order 4 with an error estimate computed from a 5th order RK method. In practise, even if the local error estimate (17) holds only for the lower order method it is customary in practise to use values given by the higher order method as the approximation of the solution at grid point $s_{k}$ since they are more accurate. This is sometimes interpreted as local extrapolation. While in such a case relation (17) is still used for step-size control purposes it is no longer related asymptotically to the local error for the propagated solution. One can report that there exists some ERK methods such as the method of Dormand and Prince [22] that are designed to minimise the local error of the higher order solution.

Last, one has to point out that the main idea behind the concept of ERK method is to have a large part of the internal computations of the $2 \mathrm{RK}$ schemes in common in order to have a computational cost much lower than the one required when using 2 arbitrary RK methods of order $p$ and $q$.

\subsection{Embedded Runge-Kutta methods for the IP method}

When looking for an ERK method for using in conjunction with the IP method, 2 different approaches can be explored: 
- the first one would be to look for a 4th order RK method embedded in a 5 th order RK method so as to design an adaptive step-size strategy based on the estimation of the local error of the 4th order RK method;

- the second one would be to look for a 3rd order RK method embedded in a 4th order RK method and to use the local extrapolation idea to propagate the solution computed with the 4th order RK method.

The main drawback of the first approach lies in the number of computational stages required by a 5 th order RK method which is 6 at least [18]. Even if part of the stages are in common between the 2 ERK methods, this approach implies a significant extra cost of at least 2 stages. In the situation considered here, each stage of the RK method requires one evaluation of function $\mathcal{G}_{k}$ which itself involves 2 exponential operator evaluations and one evaluation of the non-linear operator $\mathcal{N}$. The excess computation may be regarded as prohibitive and we will consider here the second approach mentioned above. There is an infinite number of 3rd order RK method embedded in a 4th order RK method and requiring 4 computational stages, see [18]. Now, we have to keep in mind that the efficiency of the RK4-IP algorithm is partially due to the values of the $c_{i}$ coefficients of the RK4 scheme $\left(c_{1}=0, c_{2}=1 / 2, c_{3}=1 / 2\right.$ and $\left.c_{4}=1\right)$. These values are capitalised to reduce the number of exponential operator terms to be computed. Other choices for the values of the coefficients $c_{i}$ would lead to a larger number of exponential operator terms and therefore to an increase in the computational cost. We have to look for an embedded Runge-Kutta method with this constraint in mind. Moreover we will only consider here explicit Runge-Kutta methods because there is no reason why using implicit RK methods which are more expensive. Additionally, we will assume that the weight coefficients $b_{i}, i=1, \ldots, 4$ are nonnegative real numbers which corresponds to an usual simplification assumption in the study of RK methods in order to avoid round off error issues for the method.

\section{The ERK4(3) method}

\subsection{A 5 stage RK3 scheme}

One can show that when imposing the values $c_{1}=0, c_{2}=$ $1 / 2, c_{3}=1 / 2$ and $c_{4}=1$ for a 4 stage RK method, we can not get a 4th order RK method embedding a 3rd order one whatever the values of the coefficients $b_{i}$ and $a_{i, j}$. We either obtain $2 \mathrm{RK}$ method of order 4 which is not suited for local error estimation or a 2nd order RK method embedded in a 4th RK method such as the standard ERK4(2) method defined by the following Butcher tableau (the coefficients of the lower order RK method are depicted in gray cells)
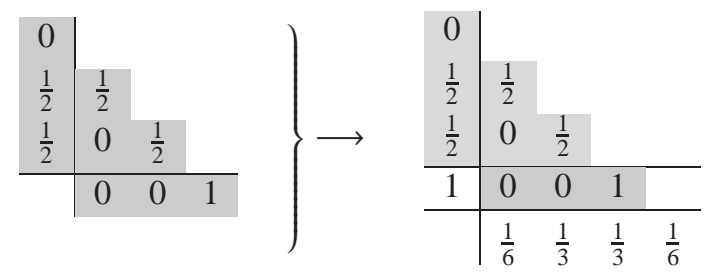

Thus the 3rd order RK method to be used together with the standard RK4 method given in (12) for local error estimation necessarily implies 5 stages. We look for a 3rd order RK method defined by a Butcher tableau in the following form:

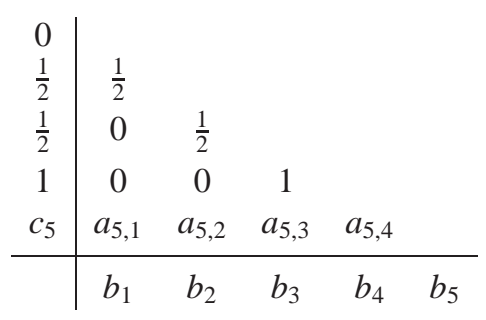

where the free coefficients $a_{5, j}$ and $b_{j}, j=1, \ldots, 4$ have to be determined in order to improve the computational efficiency of the method. It is customary for RK scheme to set the condition $c_{5}=\sum_{j=1}^{4} a_{5, j}$ and therefore the value of $c_{5}$ is imposed by the value of the other free coefficients.

The computational sequence for one step of the ERK method given in (19) applied to problem (9) becomes (see (13) and (15) for a comparison)

$$
\begin{aligned}
v_{k}^{\mathrm{ip}}(r)= & \exp \left(\frac{h_{k}}{2} \mathcal{D}\right) \cdot v_{k}^{[4]}(r) \\
\alpha_{1}= & \mathcal{G}_{k}\left(s_{k}, r, v_{k}^{\mathrm{ip}}\left(s_{k}, r\right)\right)=\exp \left(\frac{h_{k}}{2} \mathcal{D}\right) \mathcal{N}\left(v_{k}^{[4]}(r)\right) \\
\alpha_{2}= & \mathcal{G}_{k}\left(s_{k}+\frac{h_{k}}{2}, r, v_{k}^{\mathrm{ip}}\left(s_{k}, r\right)+\frac{h_{k}}{2} \alpha_{1}\right)=\mathcal{N}\left(v_{k}^{\mathrm{ip}}\left(s_{k}, r\right)+\frac{h_{k}}{2} \alpha_{1}\right) \\
\alpha_{3}= & \mathcal{G}_{k}\left(s_{k}+\frac{h_{k}}{2}, r, v_{k}^{\mathrm{ip}}\left(s_{k}, r\right)+\frac{h_{k}}{2} \alpha_{2}\right)=\mathcal{N}\left(v_{k}^{\mathrm{ip}}\left(s_{k}, r\right)+\frac{h_{k}}{2} \alpha_{2}\right) \\
\alpha_{4}= & \mathcal{G}_{k}\left(s_{k}+h_{k}, r, v_{k}^{\mathrm{ip}}\left(s_{k}, r\right)+h_{k} \alpha_{3}\right) \\
= & \exp \left(-\frac{h_{k}}{2} \mathcal{D}\right) \cdot \mathcal{N}\left(\exp \left(\frac{h_{k}}{2} \mathcal{D}\right) \cdot\left[v_{k}^{\mathrm{ip}}\left(s_{k}, r\right)+h_{k} \alpha_{3}\right]\right) \\
\alpha_{5}= & \mathcal{G}_{k}\left(s_{k}+c_{5} h_{k}, r, v_{k}^{\mathrm{ip}}\left(s_{k}, r\right)+h_{k} \sum_{j=1}^{4} \alpha_{j} a_{5, j}\right) \\
= & \exp \left(-\left(c_{5}-\frac{1}{2}\right) h_{k} \mathcal{D}\right) \cdot \mathcal{N}\left(\operatorname { e x p } ( ( c _ { 5 } - \frac { 1 } { 2 } ) h _ { k } \mathcal { D } ) \cdot \left[v_{k}^{\mathrm{ip}}\left(s_{k}, r\right)\right.\right. \\
& \left.\left.+h_{k} \sum_{j=1}^{4} \alpha_{j} a_{5, j}\right]\right)
\end{aligned}
$$

and the RK3 and RK4 approximations to the solution to problem (9) at grid point $s_{k}$ are given by

$$
\begin{aligned}
& v_{k+1}^{[3]}(r)=\exp \left(\frac{h_{k}}{2} \mathcal{D}\right) \cdot\left[v_{k}^{\mathrm{ip}}(r)+h_{k} \sum_{j=1}^{5} b_{j} \alpha_{j}\right] \\
& v_{k+1}^{[4]}(r)=\exp \left(\frac{h_{k}}{2} \mathcal{D}\right) \cdot\left[v_{k}^{\mathrm{ip}}(r)+\frac{h_{k}}{6}\left(\alpha_{1}+2 \alpha_{2}+2 \alpha_{3}+\alpha_{4}\right)\right] .
\end{aligned}
$$

By choosing $a_{5,1}=\frac{1}{6}, a_{5,2}=\frac{1}{3}, a_{5,3}=\frac{1}{3}$ and $a_{5,4}=\frac{1}{6}$ and $c_{5}=$ $\sum_{j=1}^{4} a_{5, j}=1$, the same term appears above in the expression of $\alpha_{5}$ as well as in the expression of $v_{k+1}^{[4]}$ and this can be capitalized in order to reduce the computations. ERK methods having this feature are said to satisfy the FSAL property (First Step At Last) [18]. Thus it remains to determine the values of the $b_{j}, j=$ $1, \ldots, 5$ in order that the RK method (19) has order 3 (but not order 4 ). The 4 condition equations for the method to be of the 
3rd order read [18, 23]:

$$
\left\{\begin{aligned}
b_{1}+b_{2}+b_{3}+b_{4}+b_{5} & =1 \\
\frac{1}{2} b_{2}+\frac{1}{2} b_{3}+b_{4}+b_{5} & =\frac{1}{2} \\
\frac{1}{4} b_{2}+\frac{1}{4} b_{3}+b_{4}+b_{5} & =\frac{1}{3} \\
\frac{1}{4} b_{3}+\frac{1}{2} b_{4}+\frac{1}{2} b_{5} & =\frac{1}{6}
\end{aligned}\right. \text {. }
$$

The solution $\left(b_{1}, b_{2}, b_{3}, b_{4}, b_{5}\right)$ to this under-determined linear system is $\left(1 / 6,1 / 3,1 / 3,1 / 6-b_{5}, b_{5}\right)$ where the free parameter $b_{5}$ can be arbitrarily chosen. One can check that whatever the value of $b_{5}$ the set of condition equations for the fourth order is not satisfied by the RK method (19) and therefore the method is not of order 4. Finally, we have obtained a family of 3rd order RK methods embedding the standard 4th order RK method (12) and indexed by a free parameter $\lambda$. This family is given by the following Butcher tableau

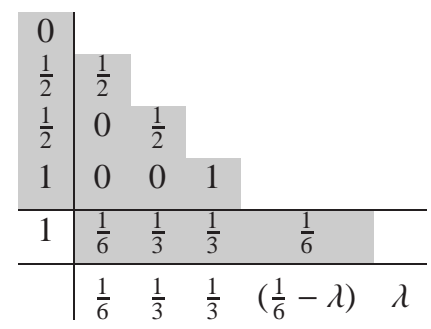

where the gray cells correspond to Butcher tableau for the standard RK4 method and the whole array is the Butcher tableau for the RK3 method. This embedded RK4(3) method actually coincides with Dormand and Prince Runge-Kutta 4(3) $T$ method [24]. Suitable value for $\lambda$ suggested in [24] is $\lambda=\frac{1}{10}$.

\subsection{Computational cost of the RK4(3) method}

The computational sequence (20) for one step of the ERK4(3) scheme defined in (22) can be improved as follows. As before one can save the computation of the $\exp \left(-\frac{h_{k}}{2} \mathcal{D}\right)$ term involved in the expression of $\alpha_{4}$ and $\alpha_{5}$ since a cancellation happens with the $\exp \left(\frac{h_{k}}{2} \mathcal{D}\right)$ term in the expression of $v_{k+1}^{[3]}$ and $v_{k+1}^{[4]}$. Moreover we recall that although the ERK4(3) method appears as a 5 stages method, its effective cost is very similar to the one of a 4 stages method since the computation of the first coefficient $\alpha_{1}$ at step $k+1$ shares the evaluation of the non-linear operator $\mathcal{N}$ in common with the coefficient $\alpha_{5}$ computed at step $k$. Namely, the computational procedure (20) can be recast as follows

$$
\begin{aligned}
v_{k}^{\mathrm{ip}}(r) & =\exp \left(\frac{h_{k}}{2} \mathcal{D}\right) \cdot v_{k}^{[4]}(r) \\
\alpha_{1} & =\exp \left(\frac{h_{k}}{2} \mathcal{D}\right) \alpha_{5, k}^{\prime} \\
\alpha_{2} & =\mathcal{N}\left(v_{k}^{\mathrm{ip}}\left(s_{k}, r\right)+\frac{h_{k}}{2} \alpha_{1}\right) \\
\alpha_{3} & =\mathcal{N}\left(v_{k}^{\mathrm{ip}}\left(s_{k}, r\right)+\frac{h_{k}}{2} \alpha_{2}\right) \\
\alpha_{4}^{\prime} & =\mathcal{N}\left(\exp \left(\frac{h_{k}}{2} \mathcal{D}\right) \cdot\left[v_{k}^{\mathrm{ip}}\left(s_{k}, r\right)+h_{k} \alpha_{3}\right]\right) \\
\beta(r) & =\exp \left(\frac{h_{k}}{2} \mathcal{D}\right) \cdot\left[v_{k}^{\mathrm{ip}}(r)+\frac{h_{k}}{6}\left(\alpha_{1}+2 \alpha_{2}+2 \alpha_{3}\right)\right] \\
v_{k+1}^{[4]}(r) & =\beta(r)+\frac{h_{k}}{6} \alpha_{4}^{\prime} \\
\alpha_{5, k+1}^{\prime} & =\mathcal{N}\left(v_{k+1}^{[4]}\right) \\
v_{k+1}^{[3]}(r) & =\beta(r)+\frac{h_{k}}{30}\left(2 \alpha_{4}^{\prime}+3 \alpha_{5, k+1}^{\prime}\right)
\end{aligned}
$$

Compared to the computational procedure (15) of the standard RK4 scheme for the IP method, the propound computational procedure (23) has a very similar computational cost even if the ERK4(3) scheme has 5 stages: the number of evaluations of the non-linear operator $\mathcal{N}$ is 4 in both cases and in both cases we have 4 evaluations of the $\exp \left(\frac{h_{k}}{2} \mathcal{D}\right)$ operator. The extra cost is limited to 2 additions and 3 multiplications and the need to keep in memory 2 intermediate results.

\subsection{Local error estimate for the ERK4-IP method}

We consider the ERK4(3) method given by Butcher tableau (22). The local errors at grid point $s_{k+1}$ for the RK3 and the RK4 schemes are respectively given by [18]

$$
\begin{aligned}
& \ell_{k+1}^{[3]}(r)=u\left(s_{k+1}, r\right)-v_{k+1}^{[3]}(r)=\psi_{3}\left(s_{k}, r, v_{k}^{[3]}\right) h_{k}^{4}+\varnothing\left(h_{k}^{5}\right) \\
& \ell_{k+1}^{[4]}(r)=u\left(s_{k+1}, r\right)-v_{k+1}^{[4]}(r)=\psi_{4}\left(s_{k}, r, v_{k}^{[4]}\right) h_{k}^{5}+\varnothing\left(h_{k}^{6}\right)
\end{aligned}
$$

where $\psi_{3}$ and $\psi_{4}$ are functions of the elementary differential of order 3 and 4 respectively. By difference of these 2 relations we obtain

$$
v_{k+1}^{[4]}(r)-v_{k+1}^{[3]}(r)=\psi_{3}\left(s_{k}, r, v_{k}^{[3]}\right) h_{k}^{4}+\varnothing\left(h_{k}^{5}\right) .
$$

Thus the local error for the 3rd order RK method at grid point $s_{k+1}$ can be approximated, with an error in $\varnothing\left(h_{k}^{5}\right)$, in the following way:

$$
\ell_{k+1}^{[3]}(r)=\psi_{3}\left(s_{k}, r, v_{k}^{[3]}\right) h_{k}^{4}+\varnothing\left(h_{k}^{5}\right) \approx v_{k+1}^{[4]}(r)-v_{k+1}^{[3]}(r) .
$$

The $\mathbb{L}^{2}$-local error at grid point $s_{k+1}$ is then computed as follows

$$
L_{k+1}^{[3]}=\left\|\ell_{k+1}^{[3]}\right\|_{\mathbb{L}^{2}(\Omega, \mathbb{C})} \approx\left(\int_{\Omega}\left|v_{k+1}^{[4]}(r)-v_{k+1}^{[3]}(r)\right|^{2} \mathrm{~d} r\right)^{\frac{1}{2}}
$$

where the integral is computed by means of a quadrature formula (e.g. the rectangle quadrature rule for its simplicity). As mentioned before, even if the local error estimate (25) holds only for the 3rd order method, in practise we use the value given by the 4th order method as the approximation of the solution at grid point $s_{k+1}$. In general, this approach overestimates the actual local error, which is safe but not of optimal efficiency. 


\subsection{Step-size control}

For step-size control, a tolerance "tol" is given as bound on the local error estimate. A step-size control strategy [18] consists in rejecting the current step-size if it gives an estimated local error higher than the specified tolerance and in accepting the solution computed with this step-size if otherwise. When the current step-size is rejected, a new smaller step-size has to be chosen to recompute the solution from the current grid point. On the contrary, when the current step-size meets the tolerance requirement for the local error it has to be scaled up for the next step computations. In both cases, the new step-size has to be estimated using the available information from the previous step computations. We consider the ERK4(3) method defined by (22) and we assume that the leading term in the asymptotic expansion (25) of the local error dominates the others for the current value of the step-size $h_{k}$. From (25) and (26) there exists $C \in \mathbb{R}^{+}$such that

$$
L_{k+1}^{[3]}=\left\|\ell_{k+1}^{[3]}\right\|_{\mathbb{L}^{2}(\Omega, \mathbb{C})}=C h_{k}^{4} .
$$

The optimal step-size $h_{\mathrm{opt}}$ is the one for which the local error estimate $L_{k+1}^{[3]}$ is the closest to the prescribed tolerance tol, i.e. $C h_{\mathrm{opt}}^{4}=$ tol. By eliminating the constant $C$ from these 2 relations we obtain

$$
h_{\mathrm{opt}}=h_{k} \sqrt[4]{\frac{\mathrm{tol}}{L_{k+1}^{[3]}}} .
$$

For robustness the step-size control has to be designed in order to respond as smoothly as possible with real or apparent abrupt changes in behaviour. This means that the step-size should not vary from one step to the other by an excessive ratio. That is the reason why we impose that the new step-size does not exceed 2 times the current step-size above and half the current stepsize below. Following these requirements, we use the following step-size control formula

$$
h_{\text {new }}=\max \left(0.5, \min \left(2.0, \sqrt[4]{\frac{\text { tol }}{L_{k+1}^{[3]}}}\right)\right) h_{k}
$$

where "tol" denotes the tolerance value specified by the user as a bound on the local error and $L_{k+1}^{[3]}$ is estimated by (26). The constant values 0.5 and 2.0 are somewhat arbitrary and have to be regarded as design parameters. Sometimes an additional safety factor (typically a value such 0.9 ) is introduced in formula (27) but it is omitted here since the method already overestimates the local error.

\section{Numerical experiments}

In the framework of a project on the numerical simulation of incoherent optical wave propagation in non-linear fibers [14] we have implemented the ERK4(3)-IP method for solving the GNLS problem (2). We present in this section numerical results from the ERK4(3) method on 2 selected applications in optics: the propagation of optical solitons and the propagation of a picosecond pulse into a single-mode fiber where fiber losses, nonlinear Raman and Kerr effects and high order chromatic dispersion are taken into account. In both cases, the adaptive stepsize strategy using the ERK4(3) method is compared to the one based on the step-doubling (SD) approach.

\subsection{Local error estimation by step doubling}

The idea behind the step doubling method (also known as Richardson extrapolation method) for estimation of the local error is the following [16]. The local error $\ell_{k+1}^{[4]}$ for the RK4 method (12) at grid point $s_{k+1}$ is given by (24). Let $v_{k+1}$ be the solution at grid point $s_{k+1}$ computed from grid point $s_{k}$ using one step of size $h_{k}$, and let $\widetilde{v}_{k+1}$ be the solution computed using two steps of size $h_{k} / 2$, in both cases assuming the solution at grid point $s_{k}$ to be exact (since we are interested in the local error). The local error at grid point $s_{k+1}$ can be approximated, with an error behaviour in $\varnothing\left(h_{k}^{6}\right)$, in the following way: $\forall r \in \Omega$

$$
\ell_{k+1}^{[4]}(r) \approx \frac{2^{4}-1}{2^{4}}\left(\widetilde{v}_{k+1}(r)-v_{k+1}(r)\right)
$$

The $\mathbb{L}^{2}$-local error at grid point $s_{k+1}$ is then approximated by

$$
L_{k+1}^{[4]} \approx \frac{2^{4}-1}{2^{4}}\left(\int_{\Omega} \widetilde{v}_{k+1}(r)-\left.v_{k+1}(r)\right|^{2} \mathrm{~d} r\right)^{\frac{1}{2}}
$$

where the integral is computed by mean of a quadrature formula. Relation (28) gives an approximation of the local error corresponding to the solution computed over the coarse grid. However, since the fine mesh grid solution is a better approximation of the solution in practise it is kept as the approximate solution. The cost of estimating the local error by the SD method is then the cost of the computation of the coarse mesh grid solution and this cost is approximately half the cost of the computation of the fine mesh grid solution since the step-size is twice larger. Thus, estimating the local error using the SD method is liable for an extra computational cost of $50 \%$ more than the cost of the computation of the approximate solution itself. Following the outline given in section 4.4, the step-size control formula when using the SD method is

$$
h_{\text {new }}=\max \left(0.5, \min \left(2.0,0.9 \sqrt[5]{\frac{\text { tol }}{L_{k+1}^{[4]}}}\right)\right) h_{k} \text {. }
$$

\subsection{Soliton solution to the NLSE in optics}

We first consider the case of the non-linear Schrödinger equation (NLSE) in optics, a simplified version of the GNLSE (2) where $\alpha=0, f_{R}=0, n_{\max }=2$. The linear operator is $\mathcal{D}: A \mapsto \mathrm{i} \beta_{2} \partial_{t t} A$ and the non-linear operator is $\mathcal{N}: A \mapsto$ $\mathrm{i} \gamma A(z, t)|A(z, t)|^{2}$. When $\beta_{2}<0$, there exists an exact solution to the NLSE known as the optical soliton [20]. Namely, if the source term is given by

$$
\forall t \in \mathbb{R} \quad a_{0}(t)=\frac{N}{\sqrt{\gamma L_{D}}} \frac{1}{\cosh \left(t / T_{0}\right)}
$$


where $N=1$ is the soliton order, $T_{0}$ is the pulse half-width and $L_{D}=-T_{0}^{2} / \beta_{2}$ is the dispersion length then the solution to the NLSE reads $\forall z \in[0, L]$

$$
\forall t \in \mathbb{R} \quad A(z, t)=\frac{N}{\sqrt{\gamma L_{D}}} \frac{\mathrm{e}^{\mathrm{i} z / 2 L_{D}}}{\cosh \left(t / T_{0}\right)} .
$$

Furthermore, for $N \in \mathbb{N}, N \geq 2$, relation (32) gives the solution to the NLSE in position $z$ multiple of $\frac{\pi}{2} L_{D}$.

Fundamental soliton $(N=1)$ doesn't provide a well suited example for exploring the features of the ERK4(3) method and for comparison purposes since its shape doesn't change on propagation. We therefore consider in the following a 3rd order soliton $(N=3)$. In Fig. 1 we show for the 3rd order soliton the adjustment of the step-size when using the ERK4(3) method for evaluating the local error with a tolerance set to tol $=10^{-6}$ and an initial step-size of $h=1 \mathrm{~m}$. The other physical parameters of the numerical experiment are $L=637.21 \mathrm{~m}, \gamma=4.3 \mathrm{~W}^{-1} \mathrm{~km}^{-1}$, $\beta_{2}=-19.83 \mathrm{ps}^{2} \mathrm{~km}^{-1}, T_{0}=2.8365 \mathrm{ps}$. The number of discretisation steps along the fiber is found to be 605 and the computation time is $69 \mathrm{~s}$ on a Intel Core 2 Quad Q6600. At the fiber end $(z=L)$, the relative global error measured with the quadratic norm is $1.1210^{-4}$ whereas the maximum relative error is $1.8910^{-4}$.

The same accuracy with a constant step-size computation would have required a step size of $0.01 \mathrm{~m}$ for a total number of step of 63722 and a computation CPU time of $5490 \mathrm{~s}$.

For comparison, when using an adaptive step-size strategy based on the SD approach with the same values of tolerance and initial step-size, we obtain that the number of discretisation steps along the fiber is 396 (or 792 if we consider that it is the accurate solution computed over the fine grid of step-size $h_{k} / 2$ that is propagated) and the computation time is $148 \mathrm{~s}$. At the fiber end $(z=L)$, the relative quadratic error is $8.8310^{-6}$ whereas the maximum relative error is $1.4810^{-5}$. The evolution of the step-size along the fiber is depicted in Fig. 1 for a comparison with the ERK4(3) method. Now if we impose to find with the ERK4(3) method a quadratic error at the fiber end of approx. $8.8310^{-6}$ (for a comparison with the accuracy obtained with the SD method) we obtain the result by setting a tolerance of $10^{-7}$ and the number of step is 1209 whereas the CPU time is $128 \mathrm{~s}$.

This simple example illustrates the fact that the ERK4(3) method overestimates the local error as mentioned in section 4.3. The consequence is that the size of the steps are a little smaller than the one obtained with the SD method on the coarse grid and therefore that a larger number of steps is required. However, since in the ERK4(3) method the local error for each step is computed faster than in the SD method the total CPU time of the computation is much lower. The global error at the fiber end $(z=L)$ is smaller when the IP method is used in conjunction with the SD method since it is not the solution computed with the RK4 method that is propagated but the more accurate one computed with the half step-size in the SD method [16].

To be comprehensive, when using the same parameters as before (a tolerance set to $10^{-6}$ and an initial step-size set to

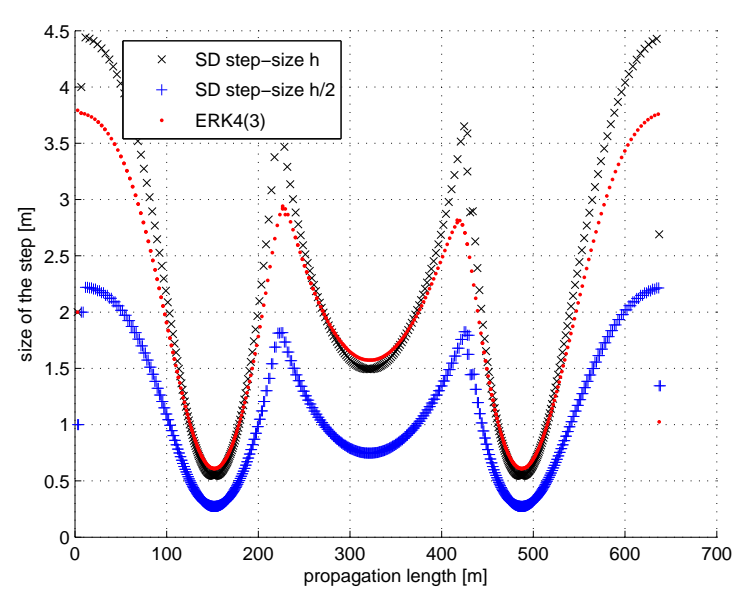

Figure 1: Evolution of the step-size along the fiber length for the ERK4(3) and the SD methods (considered over the coarse and fine grids) when solving the NLSE for a 3rd order soliton.

$h=1 \mathrm{~m}$ ) with a step-size control strategy based on the ERK4(2) method defined in (18) rather than on the ERK4(3) method, we obtain at the fiber end a quadratic relative error of $2.9610^{-8}$ and a maximum relative error of $2.7710^{-8}$. The number of discretisation steps is 4035 and the computation time is $367 \mathrm{~s}$. In this case, the local error is estimated from the solution computed with a 2 nd order RK scheme and therefore the local error is largely overestimated (since it is the more accurate solution computed with a 4th order RK scheme that is propagated) resulting in a underestimation of the optimal step-size.

\subsection{Solving the GNLSE in optics by the ERK4(3) method}

We now consider the case of the GNLSE (2) with the following set of physical parameters : $\omega_{0}=1770 \mathrm{Thz}, \gamma=$ $4.3 \mathrm{~W}^{-1} \mathrm{~km}^{-1}, \beta_{2}=19.83 \mathrm{ps}^{2} \mathrm{~km}^{-1}, \beta_{3}=0.031 \mathrm{ps}^{3} \mathrm{~km}^{-1}$ and $\beta_{n}=0$ for $n \geq 4, \alpha=0.046 \mathrm{~km}^{-1}, L=96,77 \mathrm{~m}, f_{R}=0.245$. An expression for the Raman time response function for silica core fiber is given in [20]. The Gaussian pulse at the fiber entrance $(z=0)$ is expressed as

$$
\forall t \in \mathbb{R} \quad a_{0}(t)=\sqrt{P_{0}} \mathrm{e}^{-\frac{1}{2}\left(t / T_{0}\right)^{2}}
$$

where $T_{0}=2.8365 \mathrm{ps}$ is the pulse half-width and $P_{0}=100 \mathrm{~W}$ is the pulse peak power.

In Fig. 2 we show the adjustment of the step-size when using the ERK4(3) method for evaluating the local error with a tolerance set to tol $=10^{-6}$ and an initial step size of $h=0.1 \mathrm{~m}$. The number of discretisation steps along the fiber is found to be 279 and the computation time is $50 \mathrm{~s}$. When using the SD method for determining the step-size in the IP method in the same circumstances we find that the number of discretisation steps along the fiber is 232 and the computation time is $124 \mathrm{~s}$. Here again the same comments as for the soliton case can be made when comparing the 2 adaptive step-size approaches.

When the tolerance is set to tol $=10^{-9}$ with an initial step size of $h=0.1 \mathrm{~m}$, the number of step-size with the ERK4(3) method is 1545 and the computational time is 221s whereas 906 


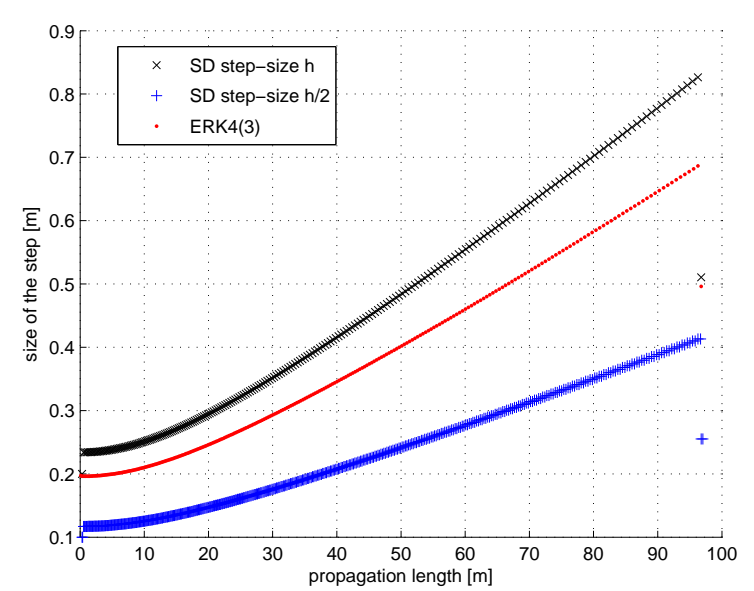

Figure 2: Evolution of the step-size along the fiber length for the ERK4(3) and the SD methods when solving the GNLSE.

steps are required by the SD method for a computational time of $645 \mathrm{~s}$. The evolution of the step-size along the fiber length is very similar to the one presented in Fig. 2.

\section{Conclusion}

We have presented an embedded Runge-Kutta scheme with orders 3 and 4 with the aim to deliver an estimation of the local error for adaptive step-size control purposes in the Interaction Picture method. The corresponding ERK4(3)-IP method preserves the features of the RK4-IP method and provide a local error estimate at no significant extra cost. When compared to the classical step-doubling approach for local error estimation and step-size control, the ERK4(3) shows a similar behaviour but a much lower computational time. As both the Symmetric Split-Step method and the IP method have a very close internal computational structure the embedded Runge-Kutta scheme could as well be used advantageously together with the Symmetric Split-Step method for local error estimation. An extension for this work would consist in looking for higher order RK schemes to be used in conjunction with the IP method. One motivation for such a study is that so as to attain a certain accuracy of the results less computational steps are required with higher RK schemes and therefore they are likely to reduce the accumulation of round-off errors. There exists in literature a lot of high order embedded RK schemes [18]. However each of these schemes has been constructed in order to satisfy one given criterion and none of them preserve the advantageous position of the internal quadrature nodes of the RK4 formula liable for the efficiency of the RK4-IP method. Therefore a next stage would be the construction of a ERK5(4) method well suited to be used in conjunction with the IP method and that preserves the ease of implementation and the advantageous position of the internal quadrature nodes of the RK4 formula so far as one can.

\section{Acknowledgments}

This work has been undertaken under the framework of the Green-Laser project and was partially supported by Conseil Régional de Bretagne, France. The authors would like to thank R. Texier-Picard from the Institute of Mathematics in Rennes, France (IRMAR CNRS UMR 6625) for her contribution to the mathematical study of the IP method as well as A. Fernandez from the Laboratoire d'Analyse et d'Architecture des Systèmes (LAAS UPR CNRS 8001) in Toulouse, France, for his major contribution to the Green-Laser project.

\section{References}

[1] B. Caradoc-Davies, Vortex dynamics in Bose-Einstein condensate, Ph.D. thesis, University of Otago (NZ), 2000.

[2] M. Davis, Dynamics in Bose-Einstein condensate, Ph.D. thesis, University of Oxford (UK), 2001.

[3] B. M. Caradoc-Davies, R. J. Ballagh, P. B. Blakie, Phys. Rev. A 62 (2000) 011602.

[4] J. Hult, J. Lightwave Technol. 25 (2007) 3770-3775.

[5] S. Wüster, T. E. Argue, C. M. Savage, Phys. Rev. A 72 (2005).

[6] R. Scott, C. Gardiner, D. Hutchinson, Laser Phys. 17 (2007) 527-532.

[7] C.-N. Liu, G. G. Krishna, M. Umetsu, S. Watanabe, Phy. Rev. A 79 (2009).

[8] J. Townsend, A modern approach to quantum mechanics, International series in pure and applied physics, University Science Books, 2000.

[9] M. Guenin, Commun. Math. Phys. 3 (1966) 120-132.

[10] R. I. McLachlan, G. R. W. Quispel, Acta Numer. 11 (2002) 341-434.

[11] G. Strang, SIAM J. Numer. Anal. 5 (1968) 506-517.

[12] J. Weideman, B. Herbst, SIAM J. Numer. Anal. 23 (1986) 485-507.

[13] C. Besse, B. Bidégaray, S. Descombes, SIAM J. Numer. Anal. 40 (2002) 26-40.

[14] A. Fernandez, S. Balac, A. Mugnier, F. Mahé, R. Texier-Picard, T. Chartier, D. Pureur, Submitted to Eur. Phys. J. - Appl. Phys. (2012).

[15] S. Balac, A. Fernandez, F. Mahé, R. Texier-Picard, Mathematical study of the Interaction Picture method for solving the nonlinear Schrodinger equation in optics, Technical Report, CNRS UMR 6082 FOTON, 2012.

[16] L. Shampine, Computing 34 (1985) 179-190.

[17] A. Heidt, J. Lightwave Technol. 27 (2009) 3984-3991.

[18] J. Butcher, Numerical methods for ordinary differential equations, John Wiley and Sons, 2008.

[19] E. Fehlberg, Classical fifth-, sixth-, seventh-, and eighth-order RungeKutta formulas with stepsize control, Technical Report, National Aeronautics and Space Administration, 1968.

[20] G. Agrawal, Nonlinear fiber optics, Academic Press, 3rd edition, 2001.

[21] A. Pazy, Semigroups of Linear Operators and Applications to Partial Differential Equations, number vol. 44 in Applied Mathematical Sciences, Springer, 1992.

[22] J. Dormand, P. Prince, J. Comput. Appl. Math. 6 (1980) 19-26.

[23] E. Hairer, S. P. Norsett, G. Wanner, Solving ordinary differential equations I: nonstiff problems, Springer-Verlag, 1993.

[24] J. R. Dormand, P. J. Prince, Celestial Mech. 18 (1978) 223-232. 